\title{
Determinação de cronograma de manutenção preventiva utilizando algoritmo genético
}

\author{
Daniel Flores \\ Departamento de Engenharia de Produção e Transportes - UFRGS \\ danflores1987@gmail.com
}

\author{
Michel J. Anzanello \\ Programa de Pós-Graduação em Engenharia de Produção - PPGEP/UFRGS \\ anzanello@producao.ufrgs.br
}

\author{
Gabriel Vidor \\ Departamento de Engenharia de Produção e Transportes - UFRGS \\ gbvidor@gmail.com
}

\section{RESUMO}

Este artigo propõe um método para otimização do cronograma de manutenção preventiva com vistas à minimização dos custos associados. Dados de falhas são coletados e modelados através de distribuições de probabilidades paramétricas. Na sequência, geram-se índices de melhoria associados aos incrementos de confiabilidade decorrentes das manutenções, valendo-se do conhecimento de especialistas de processo, conforme Tsai et al. (2001). Tais índices são integrados a formulações quantificadoras do custo incorrido pelos procedimentos de manutenção. A formulação é otimizada através de Algoritmo Genético (AG), determinando o melhor tipo de manutenção (por exemplo, manutenção total ou parcial) a ser realizada em intervalos pré-definidos. Ao ser aplicado em uma máquina de transformação de bobinas de aço plano em tubos, o método gerou um cronograma coerente de manutenções com base na avaliação de especialistas de processo, além de reduzir em $20 \%$ os custos comparados à programação empírica de manutenção. Os resultados ainda comprovam a eficiência de AGs na resolução de problemas de manutenção por conta de sua rápida convergência e fácil implementação.

Palavras-chave: Manutenção Centrada em Confiabilidade, Algoritmos genéticos.

\begin{abstract}
This paper proposes a method to optimize the preventive maintenance schedule aimed at minimizing maintenance costs. Failure data are collected and modeled by parametric probability distributions. Indices of reliability improvement due to maintenance procedures are generated based on specialists' expertise as in Tsai et al. (2001). Such indices are integrated to formulations quantifying the cost incurred by the maintenance procedures. The formulation is optimized through the Genetic Algorithm (GA), which yields the best type of maintenance (e.g., total or partial) to be performed in pre-set intervals. When applied to a flat steel tubes processing machine, the method generated a consistent maintenance schedule based on process specialists' evaluation, and reduced costs by $20 \%$ compared to the empirical maintenance schedule. In addition, results corroborated the effectiveness of GAs in solving maintenance problems given its fast convergence and easy implementation.
\end{abstract}

Keywords: Reliability Centered Maintenance, Genetic algorithms.

\section{Introdução}

A análise de dados através de técnicas quantitativas e estatísticas é fundamental para a tomada de decisão, seja gerencial ou técnica. A aplicação desse conceito nos setores de manutenção das empresas resultou na MCC - Manutenção Centrada na Confiabilidade (Reliability Centered Maintenance - RCM) (XENOS, 1998).
Juntamente com a difusão da MCC, o conceito de manutenção passou por modificações, tanto científicas quanto práticas. O setor de manutenção passou a ser observado como importante fator de competitividade, sendo tratado no nível estratégico (PINJALA, 2006). De fato, a sua direta ligação com desempenho industrial (SWANSON, 2001) e as questões emergentes, como sustentabilidade (SUPPEN et al., 2006), demandaram 
esse reposicionamento. $\mathrm{O}$ desenvolvimento de produtos também passou a incorporar preocupações de mantenabilidade após o esgotamento das ópticas financeira, de marketing e industrial como fonte de vantagem competitiva (COULIBALY, 2008). Sob o aspecto da estratégia organizacional, a maior difusão e implementação da produção enxuta trouxe consigo impacto positivo nos serviços de manutenção, ampliando a integração dos mesmos com a produção e abrindo caminho para a implantação da MCC.

Entretanto, as práticas gerenciais ainda carecem de estudos norteadores sobre como utilizar os princípios da MCC para otimização de cronogramas de manutenção preventiva. Um exemplo é a empresa estudada nesse artigo (planta de transformação de bobinas de aço plano em tubos de aço), na qual a programação de manutenção preventiva é feita de maneira empírica e pouco eficiente em termos financeiros. A literatura oferece vasta gama de abordagens para otimização de cronogramas de manutenção, como pode ser visto em Cheng et al. (2008) com a utilização de modelos de inteligência artificial, em Niu et al. (2010) na otimização de custos restritos a patamares de confiabilidade pré-definidos, e em Selvik e Aven (2011), com a inclusão de incertezas na definição do cronograma de manutenção. Tais abordagens, no entanto, geralmente estão fundamentadas em modelagens e formulações demasiadamente complexas, o que acaba inibindo sua utilização em aplicações práticas. Além disso, tais sistemáticas usualmente não consideram modalidades alternativas de manutenção (totais ou parciais, por exemplo), as quais afetam a definição do cronograma.

Este artigo propõe um método para determinação do cronograma de manutenção preventiva com vistas à minimização dos custos associados. Objetiva-se determinar o melhor tipo de manutenção (por exemplo, manutenção total ou parcial) a ser realizada em períodos pré-determinados (por exemplo, manutenção semanal). Para tanto, propõem-se modificações no método sugerido por Tsai et al. (2001); no presente artigo, dados de falhas são coletados e então modelados através de distribuições de probabilidades paramétricas (Tsai et al. (2001) valem-se de distribuição não-paramétricas). Na sequência, são gerados índices de melhoria associados aos incrementos de confiabilidade decorrentes de manutenções totais ou parciais, os quais são integrados às formulações de custo contemplando tempo de máquina parada, materiais e mão-de-obra. A formulação final é otimizada através de Algoritmo Genético (AG), o qual caracteriza-se como uma ferramenta intuitiva e de fácil implementação computacional. Ao ser aplicado em uma máquina de transformação de bobinas de aço plano em tubos, o método sugerido gerou um cronograma coerente de manutenções com base na avaliação de especialistas de processo, além de reduzir em $20 \%$ os custos comparados à programação empírica de manutenção.
O presente artigo contribui para a área de confiabilidade ao adaptar a proposição de Tsai et al. (2001), uma metodologia originalmente complexa e generalista, a uma nova sistemática mais simples e precisa. As modificações propostas dizem respeito à utilização de distribuições de probabilidades paramétricas para modelagem dos dados de falhas, ao invés da utilização de distribuições não-paramétricas (as quais são, usualmente, mais complexas e menos difundidas). O presente estudo também inova ao introduzir a avaliação subjetiva de especialistas na geração de índices de melhoria decorrentes das manutenções realizadas (no estudo de Tsai et al. (2001), tais informações provinham de dados históricos). Por fim, modificações na formulação do AG aceleram a convergência do algoritmo na obtenção de resultados de natureza discreta, quando comparado à formulação proposta por Tsai et al. (2001).

O artigo está estruturado em quatro seções, além desta introdução. A Seção 2 apresenta a fundamentação teórica em torno dos procedimentos de MCC, além de breve definição acerca de AG. A Seção 3 apresenta o método proposto para definição do cronograma de manutenção, enquanto que a Seção 4 traz os resultados da aplicação do método em uma máquina específica de uma planta de beneficiamento de aços planos. A seção 5 apresenta as conclusões e sugestões para trabalhos futuros.

\section{Referencial Teórico}

Na sequência, são descritos aspectos básicos de manutenção, confiabilidade e algoritmos genéticos para suportar as discussões realizadas no estudo.

\subsection{Manutenção}

Desde o momento em que ferramentas e equipamentos se tornaram importantes para a vida e sobrevivência do homem, surgiram atividades visando à conservação e recuperação destes itens para melhor desempenho de suas funções básicas (WYREBSKI, 1997). Tais atividades, formalmente definidas como atividades de manutenção, incluem recuperação de uma condição de desgaste, reparos ou substituições completas (LUCATELLI, 2002). Inicialmente os próprios operadores realizavam todas as atividades de manutenção e, segundo Lucatelli (2002), foi a difusão de motores elétricos no chão de fábrica que exigiu serviços de conservação especializados e dedicados. Tais serviços surgiram em 1910 (WYREBSKI, 1997) e possuíam caráter puramente corretivo (XENOS, 1998).

Com o aumento da complexidade de processos e equipamentos ao longo do século XX, surgiram novas demandas aos setores de manutenção, que se traduziram em diferentes políticas de manutenção. A primeira foi a manutenção preventiva, objetivando a prevenção de 
falhas ou quebras, mediante a criação de programas de revisões ou restaurações preventivas (WYREBSKI, 1997). A determinação do intervalo programático era feito empiricamente, após observação de dados históricos.

Outra técnica surgida foi a manutenção preventiva baseada em condição, também chamada de manutenção preditiva, baseada no monitoramento de parâmetros de funcionamento vitais para a máquina. Tais parâmetros determinam a necessidade ou não de uma intervenção de manutenção preventiva (MIRSHAWKA, 1991).

Além das citadas, existe uma estratégia de manutenção surgida a partir da implantação dos preceitos de produção enxuta, chamada Total Productive Maintenance (TPM), ou Manutenção Produtiva Total (MPT). Tem como base atividades de manutenção preventiva próativa associada a manutenções autônomas executadas pelos próprios operadores (NAKAJIMA apud CHAN et al., 2005). A Manutenção Produtiva Total utiliza como indicador de desempenho o Índice de Rendimento Operacional Global (IROG), que engloba três índices: produtividade, qualidade e utilização (LABIB apud SPERANCETTA, 2005).

O uso de modelagens matemáticas e estatísticas na determinação de comportamento das taxas de falha de equipamentos, sistemas e componentes deu origem à Manutenção Centrada em Confiabilidade (MCC). A MCC pode ser entendida como uma ferramenta para otimização de planos de manutenção visando à conservação ou restauração da capacidade operacional plena de um sistema ou equipamento (DESPHANDE; MODAK, 2002).

Complementarmente, a MCC visa decidir o que deve ser feito para que certa máquina continue realizando suas funções plenamente (MÁRQUEZ et al., 2002). $\mathrm{O}$ desenvolvimento da MCC ocorreu principalmente nos setores aeroespaciais e de defesa, posteriormente sendo adaptado a setores civis com questões críticas de segurança, como usinas nucleares (DESPHANDE; MODAK, 2002). O cerne do funcionamento do MCC está no mapeamento do sistema a ser estudado, em conjunto com as funções de risco e confiabilidade, e parâmetros como tempo médio até falha (MTTF) e tempo médio entre falhas (MTBF). Tais entradas são utilizadas para planejar a manutenção visando minimização de custos, maximização de confiabilidade ou segurança, entre outros (FOGLIATTO; RIBEIRO, 2009).

A otimização de programas de manutenção é tema recorrente na literatura, geralmente utilizando certa ferramenta ou combinando um conjunto de ferramentas para criar um método robusto. Vassiliadis e Pistikopoulos (2000) utilizaram funções de disponibilidade combinadas com funções de custo de manutenção e funções de receita a fim de otimizar a rentabilidade de um processo produtivo da indústria química. Kaegi et al. (2009) propõem a adaptação do ABM - Agent Based Modeling - como alternativa para descrição e otimização de sistemas de componentes arranjados de maneira complexa. Apleland e Aven (2000) utilizaram o Teorema de Bayes aplicado à análise de risco como base de uma metodologia de otimização de manutenção baseada em risco. O algoritmo de otimização PSO (Particle Swarm Optimization) foi utilizado por Pereira et al. (2010) para definição de cronogramas de manutenção em uma usina nuclear. Por fim, Mohanta et al. (2007) combinaram algoritmos genéticos com o algoritmo SA (Simulated Annealing) na otimização de confiabilidade e segurança de uma usina de força.

\subsection{Confiabilidade}

Confiabilidade é a capacidade de um item exercer suas funções projetadas dentro de condições declaradas durante um período de tempo especificado (FOGLIATTO; RIBEIRO, 2009). Tal capacidade pode ser descrita através de funções matemáticas, e o estudo das mesmas é chamado engenharia de confiabilidade (SALEH; MARAIS, 2006).

O crescimento dos estudos de confiabilidade desenvolveu-se após a Segunda Guerra Mundial, concentrado nas áreas aeroespacial e militar. Nesses setores, de maneira coincidente, a aplicação da confiabilidade de maneira ampla originou a MCC (FOGLIATTO; RIBEIRO, 2009; DEPHANDE; MODAK, 2002). A MCC é uma metodologia fundamentada em funções matemáticas e estatísticas. A função confiabilidade $R(t)$, também chamada de função de sobrevivência, de um certo componente ou sistema é a probabilidade de, decorrido o tempo $t$, tal sistema continuar operante, onde $T$ é a variável aleatória tempo até falha.

$R(t)=P(T>t)$

Dado que a variável de tempo até falha segue uma função acumulada $F(t)$, pode-se representar a função de confiabilidade conforme abaixo, onde $f(u)$ é a densidade de probabilidade da distribuição $F(t)$.

$R(t)=1-F(t)=\int_{t}^{+\infty} f(u) d u$

Outro conceito importante em confiabilidade é a taxa de falha, expressa pela função de risco $h(t)$, utilizada para modelar e calcular o comportamento de falhas ao longo da vida útil de um componente ou sistema de componentes (FOGLIATTO; RIBEIRO, 2009). 
$h(t)=\frac{f(t)}{R(t)}$

sujeita a $t \geq 0, h(t) \geq 0 \mathrm{e}$

$\int_{0}^{+\infty} h(t) d t=+\infty \forall \mathrm{t} \in[0,+\infty)$.

A transformação de dados de falha nas funções descritas é passo fundamental no cálculo de otimizações de manutenções centradas em confiabilidade.

\subsection{Algoritmos Genéticos}

A observação de funcionamento dos mecanismos da evolução biológica, principalmente no estudo de como a combinação de pressões seletivas com mecanismos de modificação (cruzamento, mutação) consegue resolver problemas complexos, deu origem à área de estudo denominada Algoritmos Genéticos (AG), um ramo da computação evolucionária e da inteligência artificial (TANOMARUL, 1995).

Tanomaru (1995) define AGs como "métodos computacionais de busca baseados nos mecanismos de evolução natural e na genética". Em AGs, uma população de possíveis soluções para o problema em questão evolui de acordo com operadores probabilísticos concebidos a partir de metáforas biológicas assumindo que, na média, os indivíduos representem soluções melhores à medida que o processo evolutivo continua.

Rusell e Norvig (2004) definem algoritmos genéticos como métodos estocásticos de busca formal e codificada de soluções quase-ótimas, e os estruturaram com base em cinco operadores lógicos genéticos: (i) função fitness, mede a adaptação de certa solução possível ao seu meio, ou seja, o índice de quão perto ela está da otimização desejada; (ii) seleção, seleciona soluções mais aptas para posteriores operações de formação da nova população, como clonagem e cruzamento; (iii) cruzamento, operação com objetivo de simular nos algoritmos genéticos as vantagens evolutivas trazidas pela reprodução natural; (iv) mutação, introduz um fator de aleatoriedade no algoritmo alterando cromossomos; (v) clonagem, é a duplicação direta de uma solução para a próxima geração, podendo ser explícita caso seja uma rotina declarada, ou implícita caso não existam restrições no programa que impeçam o cruzamento de pares idênticos.

Para melhor entender como os operadores genéticos se estruturam e quais seus encadeamentos lógicos, a Figura 1 apresenta o aspecto geral de um AG genérico.

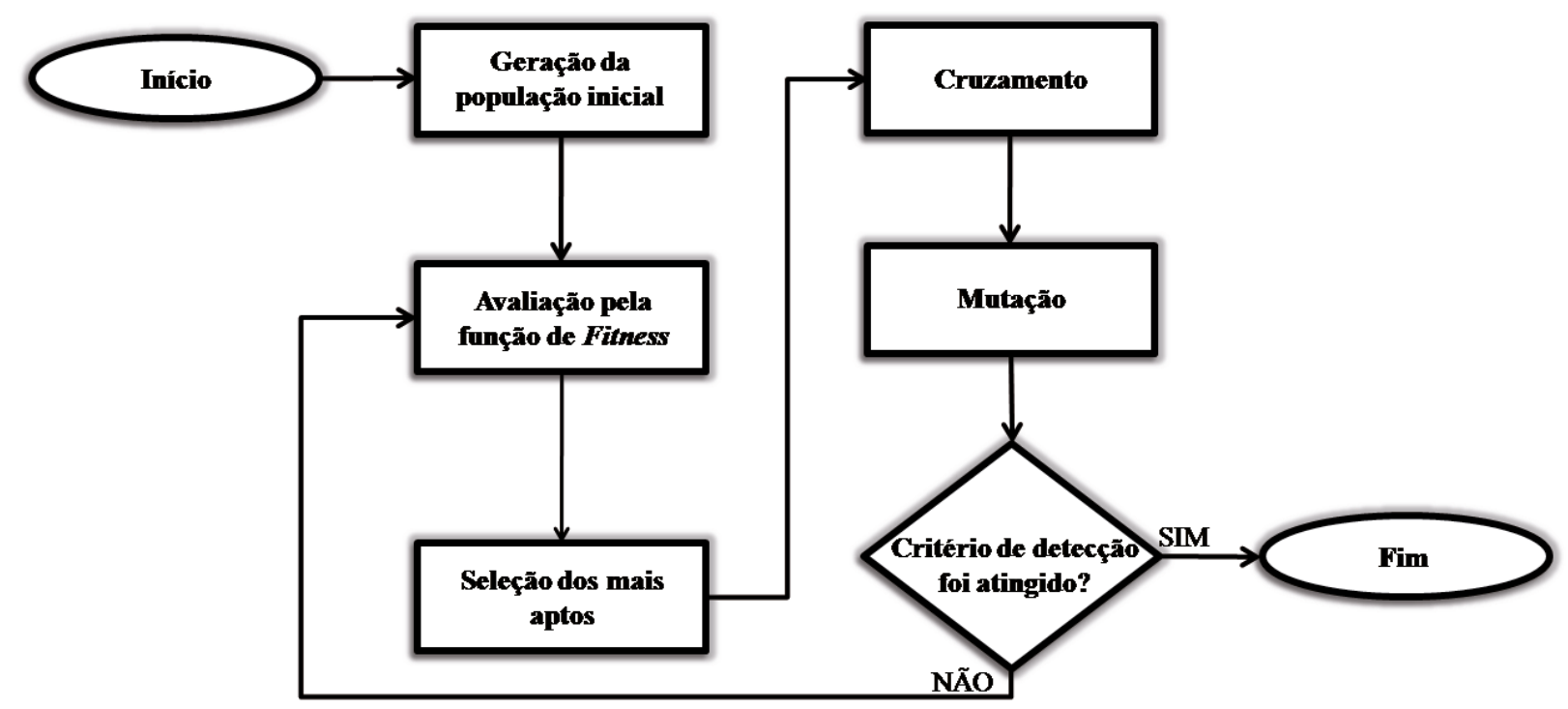

Figura 1 - Aspecto geral de um algoritmo genético

Algoritmos genéticos, em geral, são muito flexíveis, possuem grande sustentação teórica de pesquisas no campo de computação evolutiva e não requerem conhecimento teórico significativo do pesquisador em inteligência artificial, sendo necessárias somente habilidades matemáticas e de programação (RUSSELL; NORVIG, 2004). Além disso, tais métodos são robustos, pois a população inicial não tem influência, na maior parte dos casos, para impedir a obtenção de um resultado ótimo. Dentre os problemas da utilização dos AGs, destaca-se a não-garantia de encontro de ótimo global, ou seja, é possível que um algoritmo que aparentemente se comportou bem tenha atingido somente um ótimo local (GOSSELIN; POTVIN, 2009). 
Os campos de aplicação de AGs são amplos, incluindo estudos na área de otimização de manutenção. Tanomaru (1995) lista aplicações em redes neurais, análise de aglomerados (clustering) de dados, otimizações de funções de um objetivo ou multi-objetivo, controle de processos, problemas de transporte, modelos ecológicos, sociais e econômicos, máquinas de estados finitos e robótica. Carrijo (2004) aplicou a técnica na definição de regras de operação de sistemas urbanos de distribuição de água, ao passo que Gosselin e Potvin (2009) revisaram aplicações de AGs em problemas de transferência de massa e calor. Okasha e Frangopol (2009) utilizaram AGs em um problema de manutenção em sistemas estruturais, utilizando otimização multiobjetivo considerando confiabilidade, redundância e custo. Wang e Handschin (2000) propõem um novo tipo de AG inteiro com melhor eficiência que os AGs binários na resolução de problemas de manutenção de usinas de força, enquanto que Lapa et al. (2006) apresentam um modelo de plano de manutenção utilizando AGs na minimização da função de não-confiabilidade (Unreliability Function). Tsai et al. (2001) utilizou algoritmos genéticos na otimização de manutenção de componentes mecânicos com partes elétricas e comparou o uso de AGs com enumeração total das soluções, obtendo uma redução de 480 minutos para 5 minutos de cálculo em um mesmo computador. Como é possível inferir, demanda computacional também constitui-se em interesse ao utilizar-se AG.

\section{Método para Definição de Cronograma de Manutenção}

A presente pesquisa pode ser classificada como quantitativa, de natureza exploratória, em que se utilizou um caso de estudo para validar o modelo proposto. Para tanto foi realizada uma revisão da literatura, a fim de identificar abordagens para determinação de cronogramas de manutenção, optando-se pela que melhor se adaptava ao problema a ser resolvido.

Dessa forma, o método proposto parte de modificações na abordagem sugerida por Tsai et al. (2001), dado que o tipo de máquina analisada é semelhante à escolhida pelos autores, com sistema predominantemente mecânico e micro-sistemas eletroeletrônicos. O método adaptado é composto pelas seguintes etapas: (i) coleta de dados de tempo até falha e modelagem de confiabilidade utilizando aplicativo estatístico, (ii) determinação, junto a especialistas de processo, dos ganhos das manutenções preventivas para geração de um índice de melhoria decorrente dos procedimentos, (iii) determinação, junto à empresa, da confiabilidade mínima aconselhada para bom funcionamento do equipamento, (iv) levantamento de custos associados às manutenções corretivas e preventivas e formulação de uma função objetivo, (v) otimização da função objetivo via AG.

A primeira etapa consiste na coleta dos tempos até falha das máquinas analisadas. Utilizam-se ordens de manutenção cruzadas com dados de paradas de máquina, geradas pelo ERP (Enterprise Resource Planning) da empresa. Tal entrada de informações permite determinar o tempo até falha de cada sistema em operação normal. Os dados são então modelados para definir a distribuição probabilística que mais se ajusta aos mesmos, valendo-se do software de confiabilidade Proconf. São testadas as distribuições Exponencial, Weibull, Normal e Lognormal; a melhor distribuição é definida com base em testes estatísticos e papel de probabilidade.

A etapa seguinte consiste na geração de um índice $m$ para quantificação da melhoria decorrente de dois tipos de manutenção: manutenção preventiva parcial e manutenção preventiva total. Para a manutenção do tipo parcial, gera-se uma tabela relacionando tipo de atividade $\left(X_{i}\right)$ ao sistema da máquina (Tabela 1 ), adaptado do modelo proposto por Tsai et al. (2001). Tal sistemática avalia o incremento de confiabilidade gerado por uma manutenção preventiva parcial, através da avaliação do impacto de seis atividades genéricas sobre os sistemas avaliados: lubrificar, limpar, ajustar/calibrar, apertar, suprir e reparo simples. O cálculo do índice de melhoria m se dá através da equação (1), onde $p_{i j}, 0<p_{i j}<1$, é o fator de melhoria resultante da $j$-ésima atividade no $i$-ésimo sistema em uma manutenção preventiva, e $d_{i}$ é o fator de criticidade calculado em função do \% de horas paradas por conta de falhas no sistema $i$.

$m=\sum_{i=1}^{7} \frac{\sum_{j=1}^{6} p_{i j}}{d_{i}}$ 
Tabela 1 - Sistemas versus atividades de manutenção preventiva

\begin{tabular}{rccccccc}
\hline & \multicolumn{7}{c}{ ATIVIDADES - 1 a 6} \\
SISTEMAS & X1 & X2 & X3 & X4 & X5 & X6 & FATOR \\
\hline Alimentação & $p_{11}$ & $p_{12}$ & $p_{13}$ & $p_{14}$ & $p_{15}$ & $p_{16}$ & $d_{1}$ \\
Formação & $p_{21}$ & $p_{22}$ & $p_{23}$ & $p_{24}$ & $p_{25}$ & $p_{26}$ & $d_{2}$ \\
Solda & $p_{31}$ & $p_{32}$ & $p_{33}$ & $p_{34}$ & $p_{35}$ & $p_{36}$ & $d_{3}$ \\
Resfriamento & $p_{41}$ & $p_{42}$ & $p_{43}$ & $p_{44}$ & $p_{45}$ & $p_{46}$ & $d_{4}$ \\
Calibração & $p_{51}$ & $p_{52}$ & $p_{53}$ & $p_{54}$ & $p_{55}$ & $p_{56}$ & $d_{5}$ \\
Serra & $p_{61}$ & $p_{62}$ & $p_{63}$ & $p_{64}$ & $p_{65}$ & $p_{66}$ & $d_{6}$ \\
Mesa embalagem & $p_{71}$ & $p_{72}$ & $p_{73}$ & $p_{74}$ & $p_{75}$ & $p_{76}$ & $d_{7}$ \\
\hline
\end{tabular}

A manutenção preventiva do tipo total consiste na substituição completa do sistema antes de uma quebra. Como o caso estudado trata de sistemas e não componentes, a substituição preventiva é considerada como o overhaul de equipamento, consistindo na plena inspeção e revisão do sistema, com substituição de todos componentes desgastados e execução de reparos necessários. Neste caso, assume-se $m=1$.

A influência dos índices de melhoria pode ser entendida como uma retração do tempo de desgaste (uso) do equipamento, dentro de intervalos fixos e constantes de tempo.

Considere $T$ como o intervalo padrão de manutenção; se nesse intervalo não for feita nenhuma manutenção, a curva de confiabilidade avançará $T$ horas. Caso ocorra uma manutenção preventiva parcial com índice $m$, tem-se que a confiabilidade será restaurada a um nível equivalente à verificada no instante $(1-m) T$ do intervalo entre a última manutenção e o instante atual. Portanto, uma melhoria $m=1$ representaria a reposição do nível de confiabilidade verificada $T$ unidades de tempo atrás

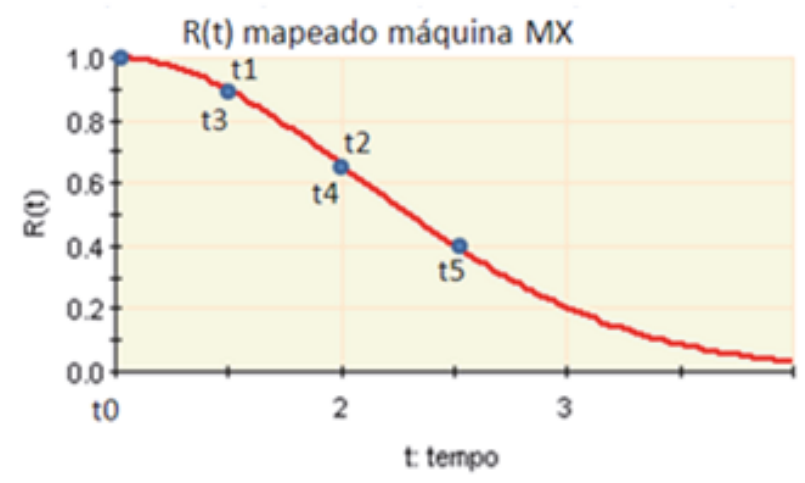

(como se não houvesse existido o período de uso $T$ em termos de redução de confiabilidade). Uma relação genérica para determinação do tempo referente à reposição do nível de confiabilidade, ou tempo de desgaste efetivo $\left(t_{i k}\right)$, é dada pela equação (2) para o $i$-ésimo componente ou subsistema ao final do $k$-ésimo intervalo, sujeito à j-ésima manutenção:

$$
t_{i k}=t_{i k-1}+T-m_{i j}\left(t_{i k-1}+T\right)
$$

onde a $j$-ésima manutenção pode assumir 3 níveis: 0 se uma manutenção não é necessária, 1 se uma manutenção preventiva parcial será efetivada, e 2 se uma manutenção preventiva total (tipo overhaul) será efetivada. Portanto, dada a função de confiabilidade do sistema da máquina $R(t)$, a confiabilidade ao final do $k$-ésimo intervalo será $R\left(t_{i k}\right)$, que equivale à $R(k T)$ para ausência completa de manutenção. O conceito proposto é ilustrado na Figura 2 , onde uma manutenção com $m=0,67$ realizada em $T$ restabelece a confiabilidade verificada no instante $0,33 T$.

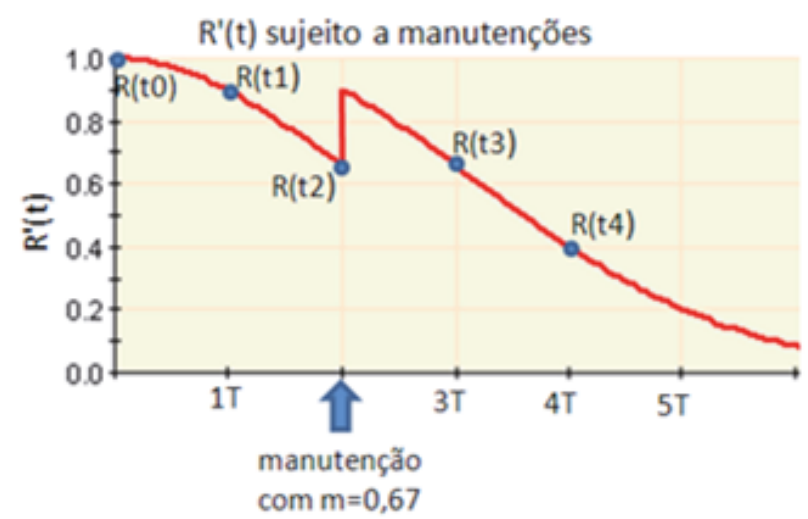

Figura 2 - Efeito do índice de melhoria de manutenção, $m$, sobre a confiabilidade

O próximo passo é a determinação da confiabilidade mínima do equipamento com base no conhecimento de especialistas de processo, levando em conta fatores de segurança e desempenho esperado. Tal confiabilidade serve como restrição à otimização. Valores de confiabilidade no intervalo $[0,85 ; 0,95]$ são usualmente utilizados em aplicações industriais (MIRSHAWKA, 1991). 
Na sequência, calcula-se o custo de manutenções corretivas do sistema, $C M C_{k}$, ao final do $k$-ésimo intervalo através da equação (3), modificada de Tsai et al. (2001). Para tanto, levantam-se os custos de cada tipo de atividade de manutenção, bem como os custos gerados por possíveis falhas do equipamento.

$$
\mathrm{CMC}_{\mathrm{k}}=\sum_{\mathrm{i}=1}^{\mathrm{n}} \sum_{\mathrm{p}=1}^{4} \mathrm{~S}_{\mathrm{pi}} \int_{\mathrm{t}_{\mathrm{ik}-1}}^{\mathrm{t}_{\mathrm{ik}}} \mathrm{h}_{\mathrm{i}}^{\prime}(\mathrm{t}) \mathrm{dt}
$$

onde $S_{p i}$ é o custo de manutenção corretiva do $i$-ésimo sistema $(i=1, \ldots, n)$ considerando $p$ fatores de custo (1: custo de parada da máquina, 2: custo de componentes substituídos, 3: custo de insumos consumidos, 4: custo hora-homem de técnicos).

Por fim, determina-se uma função objetivo (expressa em unidades monetárias) que relacione os diferentes custos de manutenção visando sua minimização ao longo de $K$ intervalos:

$$
\text { MIN } F\left(X_{1}, X_{2}, \ldots, X_{K}\right)=C M C_{k}+C M P
$$$$
\text { Sujeito a } \quad \mathrm{R}(\mathrm{t}) \geq \mathrm{R}_{\min } \forall \mathrm{t} \in\left(0, t_{K}\right)
$$

onde CMP é o custo de manutenção preventiva determinado pela soma dos custos de homem-hora e insumos consumidos por atividade. Custos de equipamento parado não são contabilizados no custo das manutenções preventivas, pois as mesmas são realizadas em momentos nos quais as máquinas estão ociosas, aos sábados.

Tal função objetivo é otimizada através de algoritmos genéticos. A solução gerada pelo AG consiste em um vetor composto pelos valores 0 (sem manutenção), 1 (manutenção preventiva parcial), e 2 (manutenção preventiva total); tais valores indicam o tipo de manutenção a ser adotada em cada intervalo $T$ ao longo de $K$ intervalos, com o mínimo custo.

O valor de $K$ é definido pelo usuário. O AG utilizado é uma adaptação do modelo proposto por Wang e Handschin (2000), com a substituição dos cromossomos binários tradicionais por valores inteiros positivos, simplificação que economiza tempo computacional.

\section{Resultados}

O método proposto foi aplicado em uma formadora de tubos de aço carbono de grande bitola e espessura, com base na análise do indicador de utilização. Foram coletados dados de falhas operacionais de janeiro a maio de 2010, período no qual a máquina operou sem qualquer tipo de manutenção preventiva. Foram coletados também dados de custos mensais médios do centro de custos individual da máquina e custos de manutenção.

Os percentuais empíricos de melhoria por conta das manutenções preventivas parciais foram obtidos junto aos especialistas de processo, gerando um fator de melhoria $m=0,2371$ [calculado de acordo com a equação (1)]. O tempo médio de parada de máquina para a execução de tais manutenções, obtido através de dados históricos, foi de 3,5 horas, contando com o trabalho de dois técnicos.

Os tempos até falha foram modelados através do software Proconf; os dados seguiram uma distribuição normal, com papel de probabilidade e parâmetros apresentados na Figura 3.

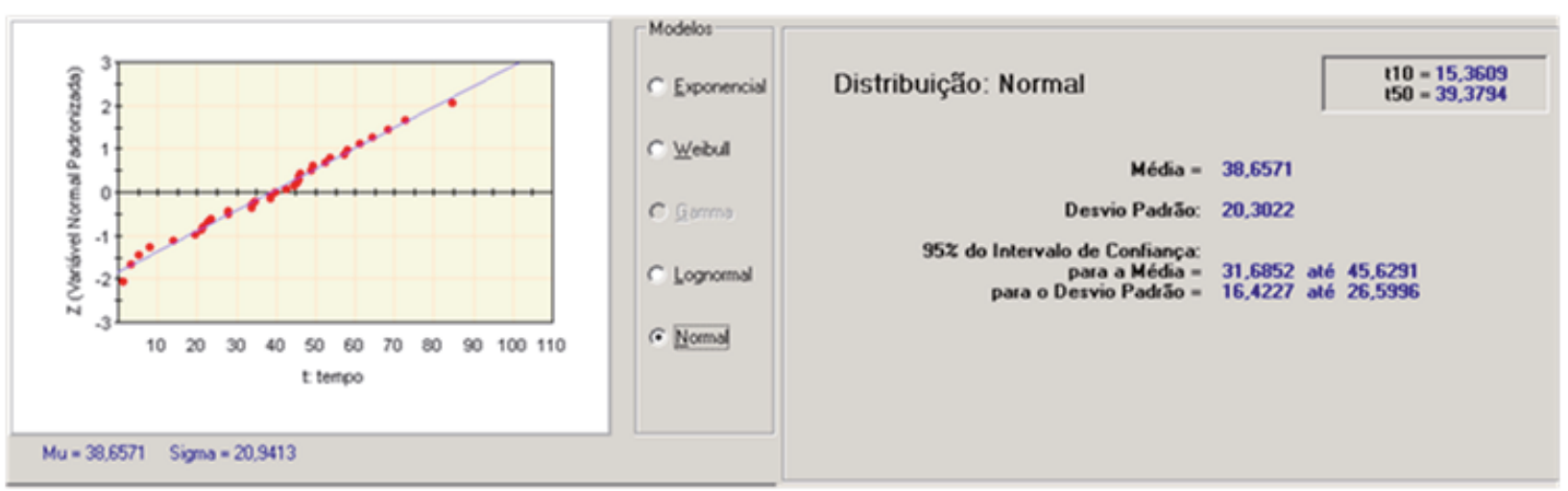

Figura 3 - Papel de probabilidade e parâmetros da distribuição normal 
Na sequência, gerou-se a função objetivo a ser minimizada, valendo-se da função de risco $h(t)$ para a distribuição normal, e custos levantados anteriormente. Considerou-se um período de manutenção de seis meses, dividido em $K=28$ semanas. A função objetivo é apresentada abaixo, considerando a $k$-ésima semana na qual foi realizada a $j$-ésima manutenção e na qual a máquina esteve sujeita aos $p$ tipos de custos preventivos e corretivos.

$$
\begin{aligned}
& \mathrm{F}\left(X_{1}, X_{2}, \ldots, X_{28}\right) \\
& =\sum_{k=1}^{28} \sum_{\mathrm{p}=1}^{4}\left(\mathrm{CMC}_{\mathrm{kp}} \int_{t_{j k-2}+T-m_{i j}\left(t_{j k-2}+T\right)}^{t_{j k-1}+T-m_{i j}\left(t_{j k-1}+T\right)} \frac{\frac{1}{\sigma \sqrt{2 \pi}} \mathrm{e}^{\frac{-(\mathrm{t}-\mu)^{2}}{2 \sigma^{2}}}}{1-\frac{1}{\sigma \sqrt{2 \pi}} \int_{-\infty}^{\mathrm{t}} \mathrm{e}^{\frac{-(\mathrm{t}-\mu)^{2}}{2 \sigma^{2}}} \mathrm{dt}} \mathrm{dt}+\mathrm{CMP}_{\mathrm{kp}}\right)
\end{aligned}
$$

\section{Sujeito a $\quad R(t) \geq 0,90$}

Salienta-se que a função taxa de falha não é integrável por método analítico, sendo necessária sua integração por métodos numéricos; utilizou-se, para isso, a quadratura adaptativa de Simpson.

Um algoritmo para o método proposto foi desenvolvido em Matlab 7.4, modificando-se a função de algoritmos genéticos apresentada no programa. As modificações focaram-se nas funções de criação e mutação da população para permitir a geração de soluções inteiras limitadas, ao invés do uso de variáveis double, de ponto flutuante. Outra alteração refere-se à função taxa de falha, programada em função separada pela necessidade de resolução de integrais por métodos numéricos. A eficiência do algoritmo foi testada para um problema piloto; resultados foram obtidos após 9 segundos de processamento, enquanto o método da enumeração total demandou 1200 segundos.

O algoritmo foi rodado com parâmetro de 250 gerações, população de 20 cromossomos e tolerância de $10^{-6}$. A otimização foi finalizada em 151 gerações, quando a mudança média na função fitness ficou abaixo da tolerância. Através da análise da evolução dos valores da função objetivo (Figura 4), percebe-se que a convergência para a solução foi rápida ao longo das gerações: o valor médio populacional da função objetivo mostra-se estável entre a geração 50 e 150, oferecendo um bom nível de confiança em sua acurácia.

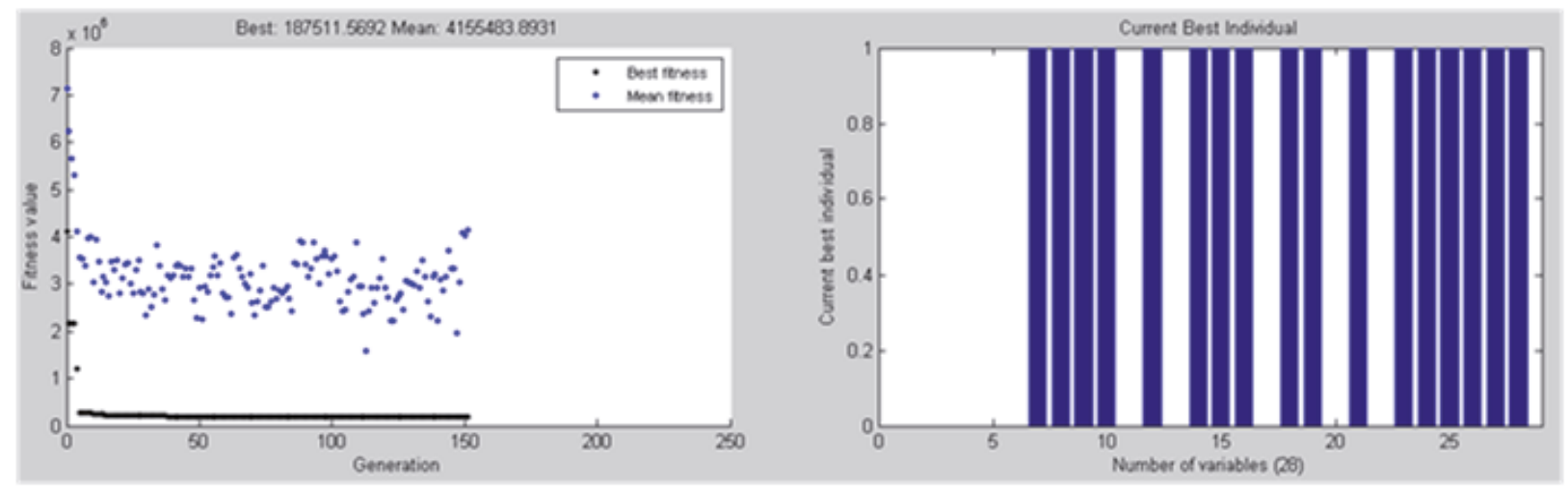

Figura 4 - Evolução gráfica da função objetivo (fitness) e solução ótima

A solução ótima ( $\mathrm{SO}$ ) encontrada, chamada cromossomo-solução, para o problema de 28 semanas de programação de manutenções foi:

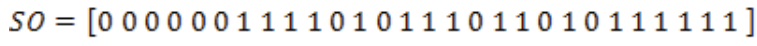

Tal solução apresenta $\mathrm{R} \$ 187.511,57$ como valor ótimo da função objetivo, no qual já estão incluídas as perdas relativas às falhas esperadas para cada período, calculadas através da integral da taxa de falha sujeita às manutenções. Verifica-se que a solução ótima não inclui manutenções do tipo preventiva total (tipo 2). Isso se dá por conta do curto horizonte considerado (seis meses), dentro do qual não se justificaria a realização de manutenções do tipo total. Tal manutenção seria provavelmente recomendada para programações 
de longo prazo, com mais de um ano de agendamento de manutenções (esse teste não é feito neste estudo).

Importante também notar que a necessidade de manutenções preventivas do tipo 1 só ocorre após 7 semanas de uso da máquina e consequente degradação de seus componentes. Manutenções antes de tal período não são justificáveis do ponto de vista de custos, pois haveria poucos componentes suficientemente desgastados para revisão e reparo/substituição. Tal resultado foi corroborado pelos especialistas da empresa.

Financeiramente, o resultado também é relevante. O custo de manutenção gerado pelo cronograma sugerido ( $\mathrm{R} \$ 187.511,57)$ é inferior ao custo médio de manutenção verificado em períodos anteriores de mesma duração, onde procedimentos foram realizados de acordo com as decisões dos especialistas de processo e somaram R $\$ 232.733,12$. Percebe-se uma economia de $\mathrm{R} \$ 45.221,55$, ou $20 \%$, para o período de 28 semanas.

\section{Conclusão}

Este artigo apresentou um método para determinação do cronograma de manutenção preventiva com vistas à minimização dos custos associados. No método proposto, baseado em Tsai et al. (2001), tempos até falha são coletados e então modelados através de distribuições de probabilidade paramétricas. Na sequência, são gerados índices de melhoria associados aos incrementos de confiabilidade decorrentes de manutenções parciais ou totais. Por fim, os índices e parâmetros oriundos das distribuições são integrados a formulações de custo de manutenção. A formulação final é otimizada através de Algoritmo Genético (AG). A aplicação do método em uma máquina de transformação de bobinas de aço plano em tubos gerou um cronograma de manutenções aprovado por especialistas de processo, além de reduzir em $20 \%$ os custos comparados à programação empírica de manutenção.

Outro resultado importante consiste na comprovação da eficiência de AGs em problemas de MCC. Tal ferramenta de otimização possibilita resolver problemas nos quais o uso de enumeração total seria proibitivo à obtenção de uma solução ótima. A baixa necessidade de tempo computacional para realizar a programação de manutenções torna viável seu uso na empresa para determinação de manutenções das principais máquinas.

Por fim, a empresa estudada apresenta fortes evidências empíricas de correlação entre velocidade de máquina e de indicadores de qualidade com a confiabilidade do equipamento. Estudos futuros podem gerar formulações voltadas à otimização da eficiência global da máquina, medida pelo IROG. A otimização de cronogramas de manutenção em sistemas multiestado, onde componentes podem falhar em múltiplos modos (ANZANELLO, 2009), também constitui-se em possível desdobramento.

\section{Referências}

ANZANELLO, M. J. A simplified approach for reliability evaluation and component allocation in three-state series and parallel systems composed of non-identical components. Gestão e Produção, v. 16, p. 54-62, 2009.

APLELAND, S.; AVEN, T. Risk based maintenance optimization: foundational issues. Reliability Engineering \& System Safety, v. 67, n. 3, p. 285-292, mar. 2000.

CAMPOS, V. F. O Significado dos Indicadores. INDG, Nova Lima, Out. 2006. Disponível em: <http:// www.indg.com.br/institucional/falconi/request_ id.asp?id=132>. Acesso em: 20 jun. 2010.

CARRIJO, I. B. Extração de regras operacionais ótimas de sistemas de distribuição de água através de algoritmos genéticos multiobjetivo e aprendizado de máquina. Tese (Doutorado) Escola de Engenharia de São Carlos, 2004.

CHAN, F. T. S.; LAU, H. C. W.; IP, R. W. L. Implementation of total productive maintenance: A case study. International Journal of Production Economics, v. 95, n. 1, p. 71-94, jan. 2005.

CHENG, Z.; JIA, X.; GAO, P.; WU, S.; WANG, J. A framework for intelligent reliability centered maintenance analysis. Reliability Engineering \& System Safety, v. 93, p. 784-792, 2008.

COULIBALY, A.; HOUSSIN, R.; MUTEL, B. Maintainability and safety indicators at design stage for mechanical products. Computers in Industry, v. 59, n. 5, p. 438-449, maio 2008.

DESPHANDE, V. S.; MODAK, J. P. Application of RCM for safety considerations in a steel plant. Reliability Engineering \& System Safety, v. 78, n. 3, p. 325334, dez. 2002.

FOGLIATTO, F. S.; RIBEIRO, J. L. D. Confiabilidade e Manutenção Industrial. Rio de Janeiro: Elsevier, 2009.

GOSSELIN, M. T.; POTVIN, F. M. Review of utilization of genetic algorithms in heat transfer problems. International Journal of Heat and Mass Transfer, v. 52, n. 9-10, p. 2169-2188, Apr. 2009.

KAEGI, M.; MOCK, R.; KRÖGER, W. Analyzing maintenance strategies by agent-based simulations: 
A feasibility study. Reliability Engineering \& System Safety, v. 94, n. 9, p. 1416-1421, Sept. 2009.

LAPA, C. M. F.; PEREIRA, C. M.; BARROS, M. P. A model for preventive maintenance planning by genetic algorithms based in cost and reliability. Reliability Engineering \& System Safety, v. 91, n. 2, p. 233-240, Feb. 2006.

LUCATELLI, M. V. Proposta de aplicação de manutenção centrada em confiabilidade em equipamentos médico-hospitalares. Tese (Doutorado em Sistemas de Informação). Programa de Pós-Graduação em Engenharia Elétrica Universidade Federal de Santa Catarina, 2002.

MIRSHAWKA, V. Manutenção Preditiva. São Paulo: Markon Books do Brasil, 1991.

MOHANTA, D. K.; SADHU, P. K.; CHAKRABARTI, R. Deterministic and stochastic approach for safety and reliability optimization of captive power plant maintenance scheduling using GA/SA-based hybrid techniques: A comparison of results. Reliability Engineering \& System Safety, v. 92, n. 2, p. 87-199, Feb. 2007.

NIU, G.; YANG, B. S.; PECHT, M. Development of an optimized condition-based maintenance system by data fusion and reliability-centered maintenance. Reliability Engineering \& System Safety, v. 95, p. 786-796, 2010.

OKASHA, N. M.; FRANGOPOL, D. M. Lifetimeoriented multi-objective optimization of structural maintenance considering system reliability, redundancy and life-cycle cost using GA. Structural Safety, n. 31, p. 460-474, 2009.

PEREIRA, M. N. A et al. A Particle Swarm Optimization (PSO) approach for non-periodic preventive maintenance scheduling programming. Progress in Nuclear Energy, v. 52, n. 8, p. 710-714, Nov. 2010.

PINJALA, S. K.; PINTELON, L.; VEREECKE, A. An empirical investigation on the relationship between business and maintenance strategies. International Journal of Production Economics, v. 104, n. 1, p. 214229, Nov. 2006.

RUSSEL, S.; NORVIG, P. Inteligência Artificial. Rio de Janeiro: Elsevier, 2004.

SALEH, J. H.; MARAIS, K. Highlights from the early (and pre-) history of reliability engineering. Reliability Engineering \& System Safety, v. 91, n. 2, p. 249-256, Feb. 2006.
SELVIK, J. T.; AVEN, T. A framework for reliability and risk centered maintenance. Reliability Engineering \& System Safety, v. 96, p. 324-331, 2011.

SPERANCETTA, A. O Impacto da Implantação do TPM nos Indicadores de Manutenção. Dissertação (Mestrado Profissionalizante em Engenharia). Escola de Engenharia. Universidade Federal do Rio Grande do Sul, Porto Alegre, 2005.

SUPPEN, N.; CARRANZA, M., HUERTA, M., HERNANDEZ, M.A. Environmental management and life cycle approaches in the Mexican mining industry. Journal of Cleaner Production, v. 14, n. 12-13, p. 1101-1115, 2006.

SWANSON, L. Linking maintenance strategies to performance. International Journal of Production Economics, v. 70, n. 3, p. 237-244, Apr. 2001.

TANOMARU, J. Motivação, fundamentos e aplicações de Algoritmos Genéticos. In: Congresso Brasileiro de Redes Neurais, 2., 1995, Curitiba. Anais... Curitiba: CNRN/Copel, p. 373-403, 1995.

TSAI, Y. T., WANG, K. S.; TENG, H. Y. Optimizing preventive maintenance for mechanical components using genetic algorithms. Reliability Engineering \& System Safety, v. 74, n. 1, p. 89-97, 2001.

VASSILIADIS, C. G.; PISTIKOPOULOS, E. N. Maintenance scheduling and process optimization under uncertainty. Computers \& Chemical Engineering. v. 25, n. 2-3, p. 217-236, Mar. 2001.

WANG, Y.; HANDSCHIN, E. A new genetic algorithm for preventive unit maintenance scheduling of power systems. International Journal of Electrical Power \& Energy Systems. v. 22, n. 5, p. 343-348, Jun. 2000.

WYREBSKI, J. Manutenção Produtiva Total - Um Modelo Adaptado. Dissertação (Mestrado em Engenharia de Produção e Sistemas). Centro Tecnológico, Universidade Federal de Santa Catarina, Florianópolis, 1997.

XENOS, H. G. D. Gerenciando a Manutenção Produtiva. Belo Horizonte: FDG, 1998.

Recebido em 30/03/2012. Aceito em 24/08/2012. 